\title{
Visuo-tactile links in covert exogenous spatial attention remap across changes in unseen hand posture
}

\author{
STEFFAN KENNETT \\ University of London, London, England \\ and University College London, London, England \\ CHARLES SPENCE \\ University of Oxford, Oxford, England \\ and \\ JON DRIVER \\ University College London, London, England
}

\begin{abstract}
We investigated the effect of unseen hand posture on cross-modal, visuo-tactile links in covert spatial attention. In Experiment 1, a spatially nonpredictive visual cue was presented to the left or right hemifield shortly before a tactile target on either hand. To examine the spatial coordinates of any crossmodal cuing, the unseen hands were either uncrossed or crossed so that the left hand lay to the right and vice versa. Tactile up/down (i.e., index finger/thumb) judgments were better on the same side of external space as the visual cue, for both crossed and uncrossed postures. Thus, which hand was advantaged by a visual cue in a particular hemifield reversed across the different unseen postures. In Experiment 2, nonpredictive tactile cues now preceded visual targets. Up/down judgments for the latter were better on the same side of external space as the tactile cue, again for both postures. These results demonstrate cross-modal links between vision and touch in exogenous covert spatial attention that remap across changes in unseen hand posture, suggesting a modulatory role for proprioception.
\end{abstract}

Mechanisms of spatial attention prioritize sensory information at attended locations, relative to other locations. Historically, in much research, spatial attention has been considered for a single modality at a time, usually vision. In daily life, however, all of our senses are typically stimulated, and information about particular objects may be available to several modalities simultaneously (as when we both touch and see an object). Therefore, researchers are increasingly considering how spatial attention may be coordinated across the different modalities.

Recent research has demonstrated cross-modal links in covert spatial attention, for both endogenous attention (e.g., Butter, Buchtel, \& Santucci, 1989; Spence \& Driver, 1996), and exogenous attention (e.g., Dufour, 1999; Kennett, Eimer, Spence, \& Driver, 2001; McDonald, Teder-Sälejärvi, Heraldez, \& Hillyard, 2001; McDonald, Teder-Sälejärvi, \& Hillyard, 2000; Spence, Nicholls,

This work was funded by the Medical Research Council (U.K.), via a program grant to J.D. and a studentship to S.K., and by a Junior Research Fellowship from St. John's College, Cambridge, to C.S. J.D. holds a Royal Society-Wolfson Research Merit Award. We thank Brian Aviss for helping to design and build the tactile cue stimulators. Correspondence concerning this article should be addressed to S. Kennett, Institute of Cognitive Neuroscience, UCL, 17, Queen Square, London WC1N3AR, England (e-mail: s.kennett@ucl.ac.uk).
Gillespie, \& Driver, 1998). Here, we are concerned with the latter case. Several behavioral studies (see Spence, 2001 , for review) have now shown that a salient but spatially nonpredictive event in one modality can produce attentional spatial cuing effects for targets in another modality (e.g., McDonald et al., 2000; Spence et al., 1998). That is, better performance is found for judging targets in one modality when presented near a preceding cue event in another modality, rather than at other locations. For instance, Spence and colleagues (Spence et al., 1998, Experiment 3) found that spatially nonpredictive tactile cues facilitate speeded choice responses to visual or auditory targets on the same side as the cue, versus on the other side. Likewise, spatially nonpredictive visual events can lead to cuing effects in speeded tactile discriminations (Spence et al., 1998, Experiment 2). Thus, cross-modal visuo-tactile links in exogenous covert spatial attention clearly exist. But the spatial coordinates (or level of spatial representation) in which such links operate remains unclear; here, we will address this issue.

In most tactile-visual cuing studies to date, participants adopted a single fixed posture throughout the experiment. Thus, tactile events on the left side of space were typically those presented to the left hand while it was placed on the left of the body, and vice versa for right tactile events. Likewise, left and right visual field events 
were typically on their respective sides of the head and body, with head posture and gaze direction fixed straight ahead. In contrast, in everyday life, our body parts adopt a frequently changing posture as we move, since we can shift our eyes, head, and hands relative to each other in innumerable ways.

Because of the fixed posture used in previous cuing studies on exogenous spatial links between vision and touch (reviewed by Spence, 2001), the existing evidence is consistent with two radically different possibilities. First, the observed cuing effects might simply reflect differential patterns of hemispheric activation, envisaged by Kinsbourne (1975) to play a major role in directing spatial attention. Thus, a visual cue in the left hemifield might advantage tactile targets on the left hand simply because both project to (and thus activate) the same contralateral (right) hemisphere, and analogously for tactile cues to the right hand advantaging visual targets in the right hemifield. The second alternative is that visuo-tactile cuing effects might reflect attention being directed within an internal representation of external space. According to this account, a cue at a particular location in one modality will benefit the same or a similar region of external space in another modality.

These two possibilities make equivalent predictions for the typical situation studied to date, in which participants adopted an uncrossed-hands posture. However, they make opposite predictions for a situation in which the hands are crossed, with the left hand now placed near the visual target in the right visual hemifield and vice versa. In terms of initial hemispheric projections, as on the Kinsbourne (1975) account, a visual cue in, say, the left hemifield should still benefit the left hand, even when that hand now lies on the right side of space. However, if exogenous cross-modal cuing effects actually arise within representations of external space, a visual cue should advantage whichever hand is currently located close to that cue in external space. Thus, a lefthemifield visual cue would benefit tactile events on the left hand for an uncrossed posture, but on the right hand for a crossed posture. Note that the latter result would reveal some remapping of the cross-modal links across changes in posture, in terms of which receptors need to be stimulated to produce the cuing advantage. It would indicate that the realignment of receptors in different modalities is taken into account for different postures, even for stimulus-driven shifts of exogenous attention, keeping those modalities in spatial register with respect to the external world.

In two experiments, we examined cross-modal spatial cuing effects between vision and touch, for both crossed and uncrossed hand postures. In Experiment 1, we examined the effects of presenting spatially nonpredictive visual cues prior to tactile targets, whereas in Experiment 2 , we examined the reverse situation. In both experiments, central fixation was required and monitored, to ensure that only covert attentional cuing effects were observed. Following Spence and Driver (1994, 1997), both experiments required a speeded up/down discrimination for each target, regardless of target side, following a nonpredictive spatial cue in the other modality on the same or the opposite side. This orthogonal cuing procedure was chosen so that both the speed and the accuracy of performance in the cued and uncued locations could be assessed. In this way, speed-accuracy tradeoffs may be discriminated from any genuine performance improvements. Furthermore, response-priming effects are precluded, since the up/down responses are equivalent for targets to the left or the right (see Spence \& Driver, 1996, 1997). In contrast, simple reaction time (RT) tasks allow only the speed to be accurately measured, whereas left/right localization tasks can be subject to response priming by a left or a right cue. Finally, a critical feature of both experiments was that the hands were always occluded from view. Hence, any effect of the manipulation of hand posture could not be due to sight of the hands but, instead, to proprioceptive signals indicating the current hand position. Any change in visuo-tactile cuing effects for the different postures could therefore indicate a role for a third modality, proprioception, in the modulation of cross-modal links in exogenous attention between two other modalities, vision and touch.

\section{EXPERIMENT 1}

On each trial in Experiment 1, a visual cue was briefly flashed on one side, while the unseen hand posture was either crossed or uncrossed (see Figure 1, left panel). The lights were irrelevant to the purely tactile task, which was to make a speeded elevation judgment of whether a subsequent tactile target stimulated an index finger or a thumb (upper vs. lower position, respectively; see Figure 1, left panel). It was equally likely that the visual cue would appear to the left or the right. The tactile target was also equally likely to appear on either the left or the right hand (and on either a thumb or an index finger), regardless of the location of the preceding, spatially nonpredictive visual cue. The two-choice up/down tactile decision was made as quickly as possible, without regard to which hand or visual field was stimulated.

Even though the visual cue was entirely irrelevant to the tactile task, we predicted, on the basis of previous work on cross-modal attention (e.g., Spence \& Driver, 1997; Spence et al., 1998), that tactile attention should be reflexively drawn toward the visual cue for a brief period. Hence, with uncrossed hands, we expected better thumb/finger discriminations for the hand on the visually cued side, rather than on the opposite side. We also sought to determine the spatial nature of any such crossmodal attention effects. In particular, when the hands were placed in a crossed posture, would the mapping between sensory receptors in the different modalities now change, to accommodate the crossed-hand positions in external space? If so, when the hands were crossed, the specific pairings of visual field stimulation and tactile stimulation that had previously led to better versus worse 


\section{Experiment 1 \\ Visual cues - tactile targets}

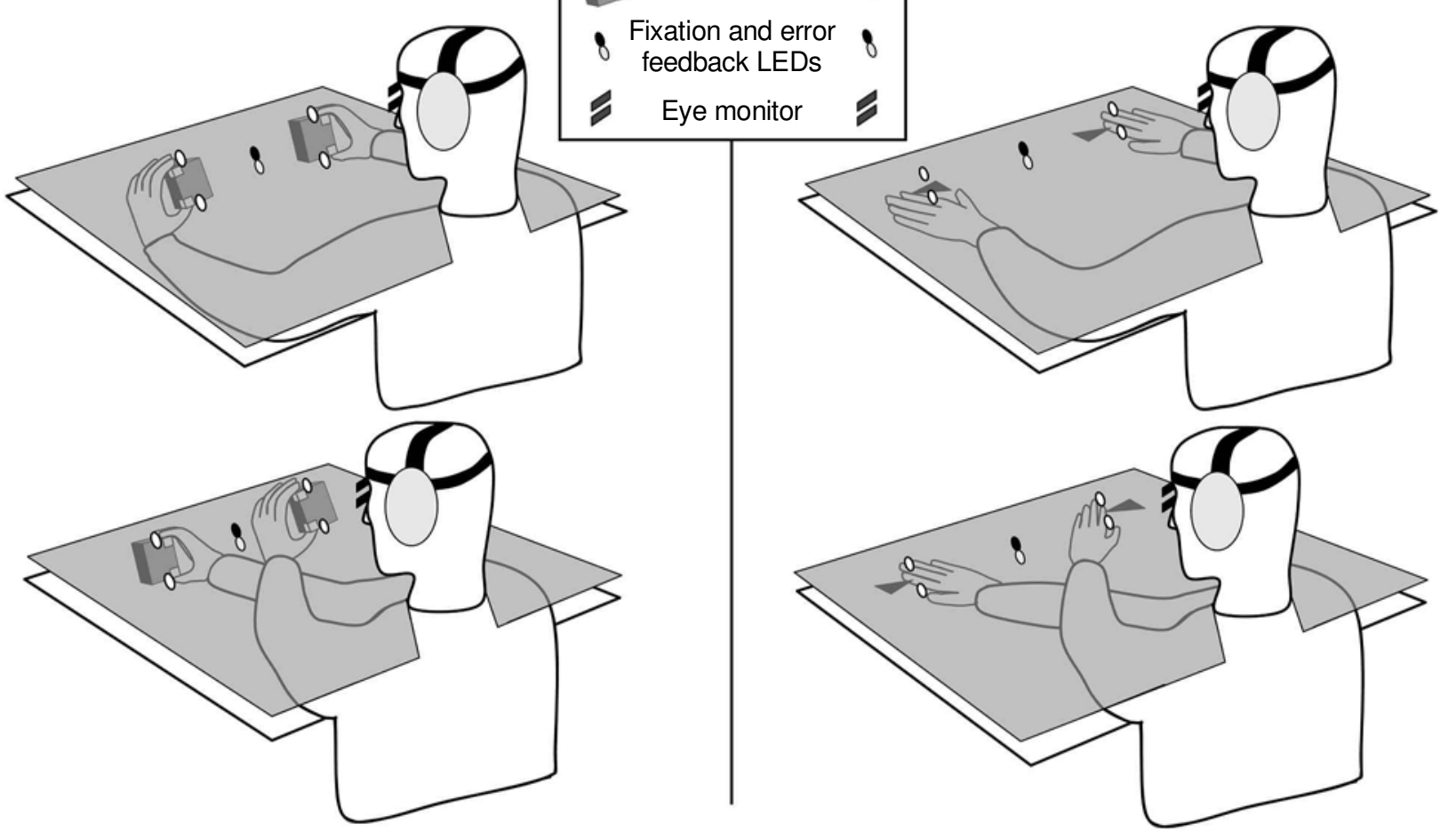

Figure 1. Schematic view of the apparatus and the participant's position for both hand postures in Experiment 1 (left), with visual cues and tactile targets, and in Experiment 2 (right), with tactile cues and visual targets. Opaque black sheets, which occluded the arms, hands, and tactile devices, are represented here in transparent gray. The participant is shown wearing an eye movement monitor to monitor central fixation and also headphones, which emitted white noise to mask the sound of the vibrotactile stimulators.

performance should now be reversed. Thus, whereas stimulation in one visual field should cue the anatomically corresponding hand when the hands were uncrossed, it should cue the other hand when they were crossed. By contrast, accounts in terms of the initial hemispheric projections for the visual and the tactile events (e.g., Kinsbourne, 1975, 1987) would predict no change in the stimulus pairings that should produce best performance, despite the change in hand posture.

\section{Method}

Participants. Twenty-four healthy volunteers (11 men and 13 women), 18-35 years of age (mean age, 26 years), were recruited by advertisement. All were naive as to the purpose of this experiment. All were right-handed and reported normal touch and normal or corrected-to-normal vision. Twelve participants performed the experiment with their hands held in an uncrossed posture, and the remaining 12 participants completed the experiment while adopting a crossed-hands posture.

Apparatus and Materials. The participants sat at able in a darkened room, with their arms resting on the tabletop, facing straight ahead. Head movements were precluded by an adjustable chinrest. A red light-emitting diode (LED) was used as a fixation point placed $480 \mathrm{~mm}$ from the participant's eyes. A yellow error feedback LED was placed immediately below this. The participant held sponge cubes between the forefinger and the thumb of each hand (see Figure 1, left panel only). Four bone conduction vibrators (Oticon-A, $100 \Omega$ ), with vibrating surfaces $16 \mathrm{~mm} \times 24 \mathrm{~mm}$, were used to present vibrotactile targets. The vibrators were driven by a 200-Hz sinewave signal (Model TE-22, sinewave generator) fed through a Realistic SA-155 amplifier, with rise and fall times of approximately $20 \mathrm{msec}$ (see Bradshaw, Howard, Pierson, Phillips, \& Bradshaw, 1992). The vibrators were placed directly on the thumb and forefinger pads for each hand. Tactile targets could be presented from any one of these four vibrators and consisted of three 40-msec pulses, each separated by A 40-msec blank interval. One red LED (diameter, $10 \mathrm{~mm}$ ) was placed directly next to each of the four vibrators, $15^{\circ}$ to the left and right of fixation. Visual cues consisted of the illumination of the two lights on either the left or the right for $90 \mathrm{msec}$. To prevent the light given off by these cue LEDs from affording any view of the current hand posture, a black sheet extended from the neck to beyond the hands, entirely covering the shoulders, arms, and hands. The cue, fixation, and error feedback lights were mounted through closely fitting holes in this sheet. White noise was presented over headphones at $75 \mathrm{~dB}$ (A) to each ear, to mask any slight noises caused by the participant's responses or by the operation of the vibrotactile stimulators.

The participants depressed two footpedals situated under the right foot throughout each block of trials: one positioned under the toes, and the other under the heel. They were instructed to raise their right heel briefly in response to lower tactile targets (thumb stimulation) and their toes in response to upper targets (index fin- 
ger stimulation). RTs were measured in milliseconds from target onset, using an 82C54 interval-timer chip on a digital input-output card (DCM-16; Blue Chip Technology, Deeside, U.K.), which interfaced to the vibrotactile stimulators, LEDs, and footpedals. The horizontal position of the left eye of all the participants was monitored (see the Procedure section for details) by means of an Eye-Trac 210 monitor (Applied Science Laboratories), which relies on the infrared scleral-reflectance method. This monitor was connected to the microcomputer controlling the experiment, via an analog-to-digital interface board (ADC-42, Blue Chip Technology).

Design. There were six equiprobable trial types produced by crossing the two within-subjects factors of cue-target stimulus onset asynchrony (SOA; 150, 200, and $300 \mathrm{msec}$ ) and side of cuing (cue same side as vs. opposite side to the subsequent target in external space). In addition, tactile targets were equally likely to appear on the left or the right and orthogonally at the index finger or the thumb, thus producing 24 subconditions. These appeared in a random order, but with equal numbers within each experimental block. There were two blocks of 30 practice trials (which were not analyzed), followed by four experimental blocks of 144 trials each. Unseen hand posture (uncrossed or crossed) was manipulated between subjects.

Procedure. The fixation light was illuminated at the beginning of each trial. The participants were instructed prior to the experiment, and periodically throughout, to maintain fixation on this light whenever it was illuminated. After an interval of $500 \mathrm{msec}$, a visual cue (two illuminated LEDs) was presented on either the left or the right for $90 \mathrm{msec}$. After a further delay (unpredictably 60, 110, or $210 \mathrm{msec}$, depending on SOA), the vibrotactile target, lasting for $200 \mathrm{msec}$, was presented from either the upper or the lower stimulator situated on the same side as the visual cue (50\% of the trials) or on the opposite side (the remaining $50 \%$ of the trials). The participants were instructed to discriminate the elevation of the tactile target as rapidly and accurately as possible by making the appropriate pedal responses with their right foot. The trial was terminated if a correct response was made. If no response was made within $1,500 \mathrm{msec}$ of target onset $(1,800 \mathrm{msec}$ in the first practice block) or if a response was made prior to target onset, both the feedback and the fixation LEDs flickered for 1,500 msec (eight 100-msec flashes, each separated by $100 \mathrm{msec}$ ). If an incorrect response was made, only the fixation LED flickered (timing as above). Following feedback, there was an additional pause of $500 \mathrm{msec}$ before trial end. After each trial, there was an interval of $600 \mathrm{msec}$ before the start of the subsequent trial. The participants were instructed to ignore the visual cues as much as possible, because they were spatially nonpredictive and, thus, unhelpful with regard to the location of subsequent tactile targets.

The eye movement monitor was calibrated to provide a signal to the computer whenever an eye movement of $3^{\circ}$ or more was detected in the period between the onset of the cue and the initiation of a manual choice response. This was the smallest signal that we could reliably detect across participants with the apparatus, but note that the tactile targets were located far from central fixation (at $15^{\circ}$ ). The monitor was recalibrated to confirm true straight-ahead at the beginning of every block and also during a block, if fixation ever appeared to drift from the calibrated center point. Trials on which potential eye movements were detected (note that blinks resulted in signals that were indistinguishable from actual eye movement signals) were automatically excluded from the analyzed data.

\section{Results}

All the participants performed the tactile discrimination task at over $85 \%$ correct. Trials were removed from the analysis if a response was given prior to target onset or if no response was made within $1,500 \mathrm{msec}$ of target onset (when the trial terminated). Fewer than $2 \%$ of all the trials were excluded by these two criteria. Trials in which the eye movement record shifted more than $\pm 3^{\circ}$ from fixation were also excluded (a further $9 \%$ of all the trials). Response errors in the up/down judgments were recorded as a percentage of remaining trials for each condition and were discarded from the RT analysis.

Participant median RTs for each of the critical conditions were calculated. The interparticipant means of these, with the respective error rates, are shown in Table 1, where "same side" and "opposite side" refer to the relative side in external space of the tactile cue and the visual target (i.e., a right visual field light after stimulation of the right hand would be same side with the hands uncrossed but would be considered opposite side with the hands crossed). Visual inspection of the data reveals that RTs were always faster for same-side cued than for opposite-side cued targets, usually with supporting trends in the accuracy data.

The data were analyzed using mixed analyses of variance (ANOVAs) on the RT and error data. The betweensubjects factor was unseen hand posture (uncrossed vs. crossed), and the two within-subjects factors were spatial cuing (visual target on the same vs. opposite side of external space as the tactile cue; i.e., same-side vs. oppositeside cue) and SOA $(150,200$, or $300 \mathrm{msec})$. In the RT analysis, there was a main effect of spatial cuing $[F(1,22)=$ $33.5, p<.001]$, caused by the participants responding more rapidly overall on same-side cue trials $(M=471 \mathrm{msec})$ than on opposite-side cue trials $(M=486 \mathrm{msec})$. There was also a main effect of SOA $[F(2,44)=15.4, p<.001]$, since the participants generally responded more rapidly to targets presented at the longer SOAs (the conventional

Table 1

Interparticipant Means of Median Reaction Times (RTs) and Percentages of Errors in Experiment 1

\begin{tabular}{|c|c|c|c|c|c|c|c|c|c|c|c|c|}
\hline \multirow[b]{3}{*}{ Measure } & \multicolumn{6}{|c|}{ Uncrossed Posture } & \multicolumn{6}{|c|}{ Crossed Posture } \\
\hline & \multicolumn{2}{|c|}{ 150-msec SOA } & \multicolumn{2}{|c|}{ 200-msec SOA } & \multicolumn{2}{|c|}{ 300-msec SOA } & \multicolumn{2}{|c|}{ 150-msec SOA } & \multicolumn{2}{|c|}{ 200-msec SOA } & \multicolumn{2}{|c|}{ 300-msec SOA } \\
\hline & $\overline{\text { Same }}$ & $\overline{\text { Opposite }}$ & $\overline{\text { Same }}$ & $\overline{\text { Opposite }}$ & $\overline{\text { Same }}$ & $\overline{\text { Opposite }}$ & $\overline{\text { Same }}$ & Opposite & Same & $\overline{\text { Opposite }}$ & $\overline{\text { Same }}$ & $\overline{\text { Opposite }}$ \\
\hline RT (msec) & 456 & 475 & 450 & 460 & 445 & 460 & 503 & 513 & 488 & 500 & 483 & 506 \\
\hline Errors $(\%)$ & 6.7 & 6.9 & 6.3 & 7.1 & 7.4 & 6.9 & 7.3 & 6.9 & 6.4 & 8.1 & 8.1 & 7.6 \\
\hline
\end{tabular}

Note-Data are broken down by relative cue-target position (same or opposite side), stimulus onset asynchrony (SOA), and posture. 
nonspatial alerting effect; e.g., Klein \& Kerr, 1974; Niemi \& Näätänen, 1981; Posner, Klein, Summers, \& Buggie, 1973). No other main effects or interactions were significant (all $F$ s $<1.3$ ).

To confirm whether spatial cuing was reliably observed not only when the arms were uncrossed, but also when they were crossed, follow-up ANOVAs were carried out on the RT data separately for each posture. The main effect of spatial cuing was significant for both postures [uncrossed, $F(1,11)=25.8, p<.001 ;$ crossed, $F(1,11)=12.5, p<.005]$. The main effect of SOA was also significant in both follow-up analyses (both $p$ s $<$ .004 ), but there was no cuing $\times$ SOA interaction (both $F$ s $<1.6)$. Thus, cuing did not reliably vary with the cue-target interval. Figure 2 (left graph) presents RTs, collapsed across SOA. The statistical significance bars shown on each bar refer to the direct pairwise comparison of same-side versus opposite-side cue trials. Note that the apparent tendency for an overall main effect of posture was not significant $[F(1,22)=1.6, p=.2]$ and that, in any case, this would reflect differences in overall RT between groups of participants and so does not address the issue of spatial cuing effects. Crucially, the spa- tial cuing effect with crossed hands shows that spatial cuing depends primarily on the relative position of the visual cue and tactile target in external space. With crossed hands, the visual cue advantaged the hand currently lying on the same side of external space, not the hand projecting to the same hemisphere. Indeed, note that if the main ANOVA had scored the data in terms of initial hemispheric projections, instead of location in external space, same-side trials would be rescored as opposite side, and vice versa, for the case of the crossed hand posture only. This means that the significant term for the main effect of cuing in the above analysis would then become a significant interaction between cuing and posture. These considerations confirm that, when assessed in terms of hemispheric projections, the results change significantly across posture, with the enhanced hand reversing when the hands are crossed.

An analogous ANOVA on the error data revealed no significant effects or interactions (all $F \mathrm{~s}<1.5$, all $p \mathrm{~s}>$ .2 ). For completeness, follow-up ANOVAs conducted separately for each posture were carried out on the error data. Neither ANOVA yielded any significant effects or interactions (all $F_{\mathrm{S}}<1.6$, all $p \mathrm{~s}>.2$ ). Overall, numeri-
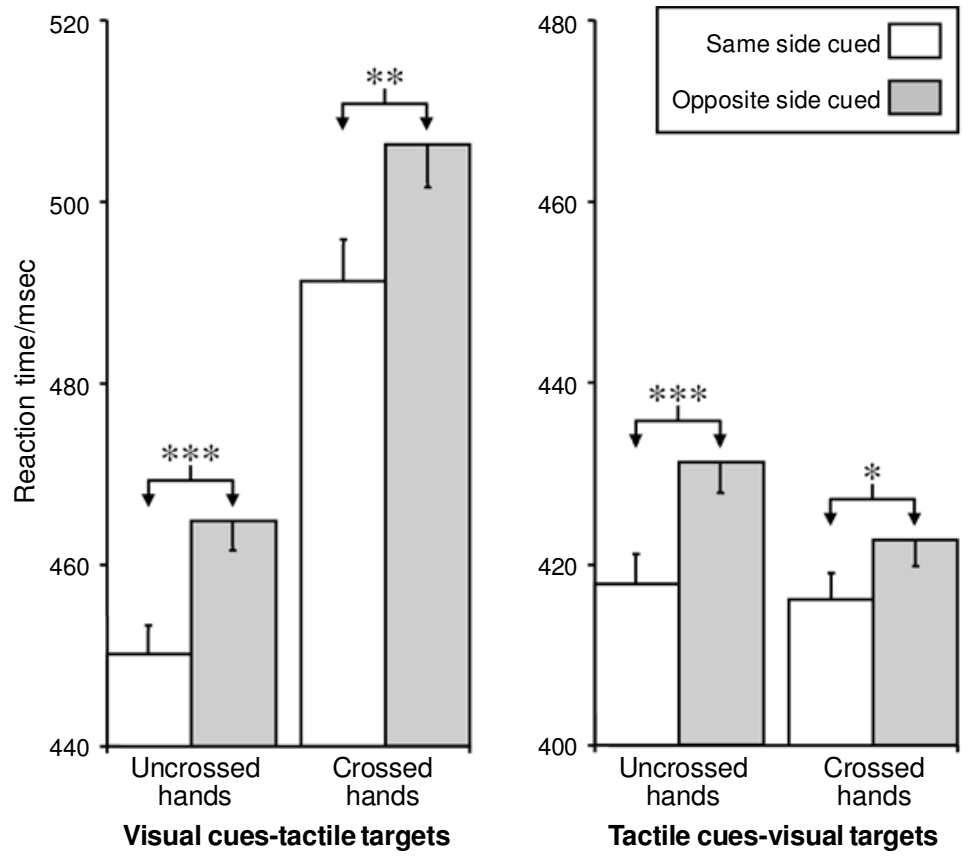

Figure 2. Interparticipant means of median reaction times from Experiment 1 (left graph) and Experiment 2 (right graph), in both uncrossed and crossed postures (collapsed across stimulus onset asynchrony). The terms "same" and "opposite" refer to the relative position of target and cue in external space. Error bars show within-subjects statistical significance bars (see Schunn, www.Irdc.pitt.edu/schunn/SSB) for the two-tailed pairwise comparison of same-side versus opposite-side cues. Note that these do not indicate significance for other comparisons. Asterisks confirm the outcome of two-tailed $t$ tests on the opposite-side versus same-side trials for each posture and in each experiment $(* p<.05 ; * * p<.005 ; * * * p<.001)$. Note that the apparent main effect of posture in the left graph is not statistically significant. 
cal trends were for more accurate performance with sameside cues than with opposite-side cues, although any such tendencies were very small (same side, 7.0\%; opposite side, $7.2 \%$ overall; see also Table 1). Thus, the RT advantages for the cued targets were not compromised by accuracy decrements but seem to reflect a genuine performance advantage for tactile targets on the same side of external space as the preceding visual cue.

\section{Discussion}

Although the visual cues were task irrelevant and spatially nonpredictive, they produced a reliable exogenous spatial cuing effect on tactile performance. Up/down (i.e., finger/thumb) judgments for tactile targets were reliably faster following a visual event on the same side of external space as the stimulated hand, versus on the opposite side, without a cost in terms of accuracy. The uncrossedhand result confirms previous reports of a cross-modal exogenous spatial cuing effect from spatially nonpredictive visual cues upon tactile targets (Spence et al., 1998, Experiment 2) and shows that this can be replicated with a different task (up/down discrimination here vs. continuous/pulsed tactile discrimination in their study) and, more important, that it can still be found with the hands unseen.

Critically, our manipulation of unseen hand posture provides new information about the spatial nature of cross-modal attentional links. When the hands were uncrossed, the same-side cue trials consisted of lights on one side prior to touch on the anatomically corresponding hand, and these trials resulted in better performance than did the reverse opposite-side combinations of lights followed by touch on the anatomically noncorresponding hand. However, when the hands were crossed, this outcome was reversed, so that it was the latter cross-modal combinations that now led to the better performance. Thus, the results changed across posture when the stimulated receptors (and initial hemispheric projections) were considered. However, they remained unchanged when considered, instead, in terms of location in external space. For both postures, a visual cue near the current location of a hand led to better tactile judgments for that hand than for the hand currently lying on the opposite side of space. Thus, the most important factor was position in external space across the modalities, not which hemisphere was initially stimulated by the projections from particularsensory receptors. Visual-tactile links, therefore, can evidently remap across changes in posture, to take into account how the current posture realigns receptors from the different modalities. Given that posture was always unseen in Experiment 1, this suggests a key role for proprioception in modulating visual-tactile links.

\section{EXPERIMENT 2}

In this experiment, issues similar to those in Experiment 1 were examined, but now with the roles of the modalities reversed. Tactile events now served as task- irrelevant, spatially nonpredictive cues, whereas the subsequent imperative target for the speeded up/down judgment was always visual. With uncrossed hands, a tactile cue on one side was expected (see Spence et al., 1998, Experiment 3) to advantage visual performance on that side, versus the other side. The novel question concerned the impact of unseen hand posture and, particularly, the pattern of any cross-modal tactile-on-visual cuing effects when the unseen hands were crossed.

In Experiment 1, tactile targets had been task relevant. The somatosensory task in that experiment might, therefore, have conceivably caused the participants to consider their current hand posture explicitly (particularly if they approached the tactile discrimination as a spatial up vs. down task, rather than merely as an anatomical finger vs. thumb discrimination). By contrast, in the present experiment, only visual events were task relevant, and all somatosensory information could simply be ignored. Indeed, the participants were now instructed to ignore all tactile events. Thus, if some remapping were to be found, similar to that observed across postures for Experiment 1, this would suggest a modulation of visuotactile links by current hand posture (signaled proprioceptively) that arises even when all somatosensory information is entirely irrelevant to the specified task.

\section{Method}

Participants. Twenty-four new healthy volunteers ( 9 men and 15 women), 19-38 years of age (mean age, 23 years), were recruited by advertisement. All were naive as to the purpose of the experiment. Twenty-two participants were right-handed, and 2 were left-handed. All reported normal touch and normal or corrected-to-normal vision.

Apparatus and Materials. The experimental layout is shown schematically in Figure 1 (right panel only). Arrangements for seating, posture, and darkness, plus occlusion of the shoulders, arms, and hands were the same as those for Experiment 1. To ensure that the tactile cues were salient, punctate tactile stimulation was now provided by a metal rod, $1.5 \mathrm{~mm}$ in diameter with a blunt end, striking the finger. The rod was propelled by a $12-\mathrm{V}$ solenoid to strike an area of the skin measuring $1.8 \mathrm{~mm}^{2}$ with a momentum of approximately 3-4 $\mathrm{gm}^{-1}$; the impression given was of a firm strike. One rod was positioned next to each hand so the rods could strike the medial surface (with the hand placed prone; i.e., palm down) of the middle segment of either index finger. Each index finger was held in place next to a stimulator with a Velcro strip.

Each hand was positioned so that the point of tactile stimulation was $530 \mathrm{~mm}$ from the participant's eyes and $25^{\circ}$ to the left or right of central fixation. The possible visual targets consisted of four green LEDs (each $5 \mathrm{~mm}$ in diameter) arranged in two vertical pairs. One pair was placed on each side, with one LED above the tactile position and the other below (as viewed from the participant's position; see Figure 3). The lights in each pair had a vertical separation of approximately $7^{\circ}$ (i.e., appearing $3.5^{\circ}$ above or below the point of possible tactile stimulation on that side). Each LED pair was placed at the same $25^{\circ}$ of visual eccentricity as one of the tactile stimulators, $50 \mathrm{~mm}$ closer to the participant's eyes. The fixation stimulus was a centrally located red LED (2-mm diameter), positioned $25^{\circ}-35^{\circ}$ (according to the height of the chinrest) below eye level. The position of the right eye was monitored using either an Iris 6500 (Skalar, The Netherlands) or an Applied Science Laboratories 210 infrared eye-tracker, calibrated for central fixation as in Experiment 1. Error feedback regarding central fixation and re- 
Participant's view

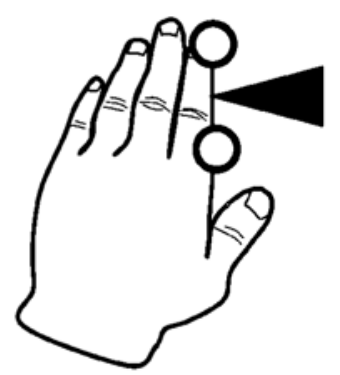

\section{Side view}

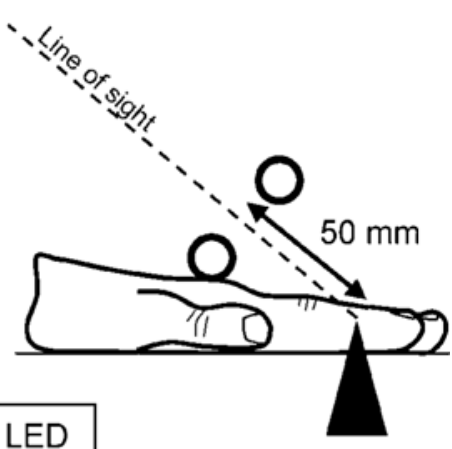

Target LED

Tactile cue

Figure 3. Diagrams showing the proximity of the tactually stimulated finger and the target lights in Experiment 2. The participant's own view (left diagram) places the tactually stimulated position halfway between the upper and the lower visual target locations. The right diagram shows the cue and target positions from the side.

sponses was provided by a yellow LED (5-mm diameter) placed immediately below fixation.

Responses were via footpedals placed under the right foot, as in Experiment 1, with analogous response mappings for the up/down task (although the targets were now visual rather than tactile). Extraneous noise was masked with white noise presented continuously through whole-ear headphones at $80 \mathrm{~dB}$ (A) to each ear.

A tactile cue consisted of three 50-msec strikes, separated from one another by $20 \mathrm{msec}$, to one or the other index finger (total duration, $190 \mathrm{msec}$ ). These salient stimuli were used as cues to maximize the likelihood of observing a robust cuing effect. Each visual target consisted of the illumination of one of the four green LEDs for $100 \mathrm{msec}$. Response error feedback to the participant was provided by the illumination of the central yellow LED for $350 \mathrm{msec}$ at the end of any trial in which the response was incorrect, occurred before target onset, or was too slow (occurring more than 2,000 msec after target onset). Excessive horizontal deviation of eye position (i.e., greater than $\pm 3^{\circ}$ ) and/or blinks were signaled at trial end, after any response error feedback, by the flashing of the same yellow LED four times ( $50 \mathrm{msec}$ for each flash, separated by $50 \mathrm{msec}$ ).

Design. Within each experimental block, there were four conditions produced by crossing the within-subjects factors of cue-target SOA (200 or $350 \mathrm{msec}$ ) and cuing (cue on same side or opposite side with respect to the subsequent target in external space). In addition, visual targets were equally likely to be on the left or the right, in an upper or a lower position, leading to 16 possible subconditions. These were presented pseudorandomly, with equal numbers within each experimental block. There was one block of 50 practice trials (which was not analyzed), followed by six experimental blocks of 96 trials each. Finally, unseen hand posture (uncrossed or crossed) was now manipulated within subjects in a blocked manner (see below). This within-subjects manipulation of posture was adopted because it should be more sensitive (Howell, 1997) to effects of the magnitude revealed by Experiment 1 than was the between-subjects procedure adopted there.

Procedure. Each trial started with illumination of the central fixation light. After a variable delay $(380-580 \mathrm{msec})$, a tactile cue was presented (for $190 \mathrm{msec}$ ) with equal likelihood to either the left or the right index finger. Following an interstimulus interval (equiprobably 10 or $160 \mathrm{msec}$ ), a single visual target was presented (thus giving two possible cue-target SOAs of 200 and $350 \mathrm{msec}$ ). This vi- sual target was equally likely to appear in any one of the four possible locations, rendering the tactile cue spatially nonpredictive. The participants were instructed to ignore the tactile cues, since they provided no helpful spatial information, and to provide only a speeded judgment of the visual target's elevation (up vs. down, regardless of target side, analogous to the tactile task in Experiment 1). As soon as a pedal response was recorded or if no response was made within 2,000 msec of target onset, the fixation light was extinguished, and any feedback was given. There was then an intertrial interval of $400 \mathrm{msec}$ before illumination of the fixation light started the next trial.

Unseen hand posture changed after each experimental block. In half of the blocks, the participants placed the left hand, with the attached tactile stimulator, behind the left pair of target lights and the right hand plus the stimulator behind the right pair of target lights (hands uncrossed; see Figure 1, top right). In the other half of blocks, the participants crossed their arms to place the right hand and stimulator behind the left visual targets and vice versa (hands crossed; see Figure 1, bottom right). The posture adopted for the practice block was counterbalanced across participants, and this posture was maintained for the first experimental block of trials.

\section{Results}

All the participants performed the speeded visual discrimination task at over $80 \%$ correct. Trials were removed from the analysis if no response was recorded, if a response was given prior to target onset, or if no response was made within $1,500 \mathrm{msec}$ of target onset (fewer than $1 \%$ of all the trials were excluded by these three criteria). Trials in which the eye position record exceeded its criterion were also excluded (a further $7 \%$ of all the trials). Response errors in the visual up/down judgments were recorded as a percentage of remaining trials for each condition and were discarded from the RT analysis.

Participant median RTs for each condition were again calculated. The interparticipant means of these, with the respective error rates, are shown in Table 2, where "same side" and "opposite side" again refer to the relative side 
Table 2

Interparticipant Means of Median Reaction Times (RTs) and Percentages of Errors in Experiment 2

\begin{tabular}{|c|c|c|c|c|c|c|c|c|}
\hline \multirow[b]{3}{*}{ Measure } & \multicolumn{4}{|c|}{ Uncrossed Posture } & \multicolumn{4}{|c|}{ Crossed Posture } \\
\hline & \multicolumn{2}{|c|}{ 200-msec SOA } & \multicolumn{2}{|c|}{ 350-msec SOA } & \multicolumn{2}{|c|}{ 200-msec SOA } & \multicolumn{2}{|c|}{ 350-msec SOA } \\
\hline & Same & Opposite & Same & Opposite & Same & Opposite & Same & Opposite \\
\hline RT (msec) & 433 & 445 & 402 & 418 & 434 & 437 & 399 & 408 \\
\hline Errors (\%) & 5.0 & 6.5 & 9.4 & 10.5 & 6.2 & 5.8 & 10.5 & 11.3 \\
\hline
\end{tabular}

Note-Data are broken down by relative cue-target position (same or opposite side), stimulus onset asynchrony (SOA), and posture.

in external space of the cue and the target. It can be seen in Table 2 that RTs were always faster for same-side cued versus opposite-side cued targets, usually with supporting trends in the accuracy data.

These observations were verified by three-way withinsubjects ANOVAs on the RT and error data from Experiment 2. The factors were spatial cuing (same side vs. opposite side, in terms of external space), SOA (200 vs. $350 \mathrm{msec}$ ), and hand posture (uncrossed vs. crossed). The RT analysis revealed a main effect of spatial cuing $[F(1,23)=20.8, p<.001]$, with visual judgments faster after same-side tactile cues $(M=417 \mathrm{msec})$ than after opposite-side cues $(M=427 \mathrm{msec}$ ) overall. (Note that, as in Experiment 1, this significant term would reflect an interaction between spatial cuing and posture if the results were rescored in terms of initial hemispheric projection, rather than external location. Such rescoring would reverse which trials are considered same-side or opposite-side cued only when the hands were crossed.) There was also a main effect of SOA $[F(1,23)=107.6$, $p<.001]$, with faster responses at the 350-msec SOA $(M=$ $407 \mathrm{msec})$ than at the $200-\mathrm{msec}$ SOA $(M=437 \mathrm{msec})$, presumably owing to nonspatial alerting factors (as in Experiment 1). The interaction of spatial cuing with hand posture was not significant $[F(1,23)=2.6, p=.12]$. Thus, although the crossed postures did yield numerically lower cuing effects than did the uncrossed posture ( mean cuing: uncrossed $=13 \mathrm{msec}$; crossed $=7 \mathrm{msec}$ ), this difference was not statistically reliable. No other terms approached significance (all $F \mathrm{~s}<1.7$, all $p \mathrm{~s}>.2$ ).

As before, to assess whether spatial cuing was reliably observed not only when the hands were uncrossed, but also when crossed, follow-up ANOVAs were carried out on the RT data, separately for the two postures. Main effects of cuing were found for both postures [uncrossed, $F(1,23)=17.0, p<.001 ;$ crossed, $F(1,23)=5.4, p=.03]$. The cuing effect with crossed hands was thus reliable, despite its relatively small size. This reliable effect reflects the importance of the relative position of the tactile cue and the visual target in external space, not merely initial hemispheric projections. The main effect of SOA was significant in both follow-up analyses (both $p$ s <.001), but there was again no interaction of cuing with SOA (both $F \mathrm{~s}<1$ ). Figure 2 (right graph) presents reaction times, collapsed across SOA, alongside the analogous data from the previous experiment (left graph). Note that any effect for which these bars do not overlap indicates reliable spatially specific cuing effects in the corresponding condition. As Figure 2 shows, cuing effects in the present experiment were stronger with uncrossed hands but were, nevertheless, significant in both postures.

The analogous error analysis revealed only a main effect of SOA $[F(1,23)=66.5, p<.001]$, with visual judgments less accurate at the 350 -msec SOA (mean error rate $=10.4 \%)$ than at the $200-\operatorname{msec}$ SOA $(M=5.9 \%)$, consistent with the usual speed-error pattern for nonspatial alerting effects (see Posner et al., 1973). No other main effects or interactions approached significance (all $F_{\mathrm{s}}<1.4$, all $p \mathrm{~s}>.25$ ).

For completeness, follow-up ANOVAs were carried out on the error data, separately for each posture. Both ANOVAs found the main effect of SOA (both $p s<.001$ ). No other significant terms were found, although the main effect of side in the uncrossed analysis approached significance, since there was a trend for more accurate performance on same-side cue trials $[F(1,23)=3.1, p=$ .09 ; all other $F \mathrm{~s}<1.1]$. As before, in both postures, the small overall numerical trend was for more accurate performance with same-side cues than with opposite-side cues, supporting the significant spatial cuing effects found in RTs (see Table 2).

\section{Discussion}

The results of Experiment 2 confirm that tactile cues can produce cross-modal spatial cuing effects on visual judgments, consistent with multimodal mechanisms of covert exogenous spatial attention (Spence et al., 1998). The critical manipulation of unseen hand posture again revealed that, as for the effects of visual cues on tactile performance in Experiment 1, tactile cuing effects on visual judgments can be determined by the location of events in external space. Thus, those pairings of tactile stimulation on one hand, together with a visual target in a particular hemifield, that had produced most efficient performance with uncrossed hands actually produced less efficient performance than did the other pairings when hands were crossed. As for Experiment 1, this implies some remapping of attentional links between touch and vision, in terms of which receptors must be stimulated to yield optimum performance. This shows that tactile-visual links in exogenous covert spatial attention take current posture into account. 


\section{GENERAL DISCUSSION}

In two experiments, we examined cross-modal links in exogenous covert attention between vision and touch. We also examined the spatial nature of any such links by manipulating unseen hand posture. We found in Experiment 1 that tactile judgments on a particular hand were enhanced by presenting a spatially nonpredictive visual cue nearby on the same side of space, versus on the opposite side near the other hand. Similarly, in Experiment 2 we found that visual judgments on one side were more efficient following a spatially nonpredictive tactile cue delivered to a hand located on that side of space. The results with uncrossed hands confirm and extend previous findings of cross-modal links in exogenous covert attention between vision and touch (see, e.g., Spence et al., 1998). But the most critical new data come from the comparison of uncrossed- and crossed-hand postures.

In both experiments, performance was faster (with small tendencies for greater accuracy) when the cue appeared at the same location in external space as the subsequent target in the other modality. This means that the particular pairings of tactile stimulation on a particular hand, together with visual stimulation in a particular hemifield, that gave the most efficient performance for one posture actually reversed across the change in unseen posture. This demonstrates that cross-modal links between vision and touch can remap across postural changes in order to take the current position of body parts into account. It argues against any explanation of tactile-visual links in exogenous attention that appeals solely to hemispheric activation, in terms of the initial hemispheric projections from each hand and each visual field.

Neither experiment found a significant relationship between spatial cuing and the cue-target interval. However, an inspection of the RT data in Tables 1 and 2 reveals that the RT advantage for the same-side versus oppositeside cued target tended to increase with SOA, particularly for the crossed-hand posture. This may point to the time course of processes that resolve spatial relationships among the modalities, particularly in the noncanonical, crossed posture adopted here. This possibility, without decisive statistical corroboration within the present experiments, requires further investigation.

Some previous work has also used the manipulation of crossing the hands. Most of this work (Bradshaw et al., 1992; Riggio, Gawryszewski, \& Umiltà, 1986; Umiltà, Rizzolatti, Anzola, Luppino, \& Porro, 1985; Wallace, 1971, 1972) was concerned with stimulus-response compatibility effects, of the type often referred to as the Simon effect (see Simon, 1990, for a review). The present experiments differ importantly from such work, because lateral responses with the hands were never required. Instead, up/down judgments were made with a unilateral pedal response, in which the elevation of lateral targets following a lateral cue was discriminated. As has previously been discussed by Spence and colleagues
(Spence \& Driver, 1994, 1997; Spence et al., 1998), this orthogonal-cuing procedure highlights attentional effects on target processing, not stimulus-response compatibility effects.

To our knowledge, only two previous studies have used a hand-crossing manipulation while seeking to study cross-modal effects of covert spatial attention. Hikosaka, Miyauchi, Takeichi, and Shimojo (1996) used the visual line-motion illusion to examine possible cross-modal links between touch and vision. In the unimodal visual version of this illusion, a horizontal line segment, which actually appears instantaneously, seems to extend from the attended end toward the other end. Hikosaka et al. reported a cross-modal variant; a tactile cue near one end of the line can apparently also induce this illusion, with its influence reportedly depending on current hand posture. Although those suggestions seem consistent with the present conclusions, Hikosaka et al.'s subjective line-motion measure has since been criticized (e.g., Downing \& Treisman, 1997; Steinman \& Steinman, 1997). Moreover, hand posture remained visible throughout Hikosaka et al.'s study, and it is unclear whether eye position was recorded, unlike in the present experiments.

More recently, the influence of hand posture on visualtactile links in endogenous covert attention has been examined (Spence, Pavani, \& Driver, 2000). Unlike the exogenous cuing effects studied here that were induced by spatially nonpredictive peripheral cues, Spence et al. (2000) examined situations in which the participants expected targets in a particular modality to appear on one prespecified side. They found cross-modal links for endogenous attention, in that expectancies in one modality had some influence on performance in the second modality also. This study differs from our own in several critical respects. First, it concerned endogenous rather than exogenous mechanisms of covert spatial attention, and these must be considered separately, given the many known differences between these types of attention, in both their psychological effects and their neural bases (e.g., Butter, 1987; Rafal, Henik, \& Smith, 1991; Robinson, Bowman, \& Kertzman, 1995; Robinson \& Kertzman, 1995). Second, hand position was not occluded in the Spence et al. (2000) study; although their experiments were conducted in a dark room, illumination from the brief visual targets would have allowed visible information about current hand position, unlike in the present study. Moreover, Wann and Ibrahim (1992) showed that even very brief glimpses of hand position, as during transient illumination, can significantly affect perceived hand location in darkness, thus confirming that Spence et al.'s (2000) setup may have been insufficient to eliminate useful visual information about posture. In the present study, vision of hand posture was eliminated not only by darkness, but also by occlusion, which completely prevented any view of the hands and arms even during illumination of a visual cue or target.

Finally, the cross-modal links were found to remap fully in Spence et al.'s (2000) endogenous study, in the 
sense that the effects with crossed hands were equivalent in size to those with uncrossed hands (although they were reversed in terms of the particular hands and visual hemifields that were affected together). Similarly, Experiment 1 here showed equivalent effect sizes in crossed and uncrossed postures (see the leftmost two bars in Figure 2). The present Experiment 2 also showed no significant differences in the size of cuing in crossed versus uncrossed postures, although the numerical size and statistical reliability of the respective effects did differ (see the rightmost two bars in Figure 2). This apparent difference between the present Experiment 1 and Experiment 2 may relate to the fact that touch was task relevant in the former experiment, but not in the latter. Attending to touch, particularly when performing a spatial tactile judgment, as for the present up/down task, may lead the current posture of the hands to be considered more fully than when all somatosensory information is entirely task irrelevant, as in Experiment 2. Note that touch was always task relevant in Spence et al.'s (2000) endogenous study, which, like the present Experiment 1, found full remapping.

Thus, the task relevance, or otherwise, of somatosensory information might determine whether remapping across changes in posture is full or partial. But the fact that hand posture still modulated the cuing effects to a significant (albeit numerically reduced) extent, even when all somatosensory information was entirely irrelevant to the prescribed visual task (as in Experiment 2), indicates that some degree of remapping can arise automatically. The present influences of hand posture were presumably due to proprioceptive signals about arm and hand location, given that the posture was unseen throughout. ${ }^{1}$ Our results thus demonstrate that a third modality (here, proprioception) can modulate attentional interactions between another two modalities (here, vision and touch) even for the case of purely exogenous shifts of covert attention. Although suggesting a rather sophisticated mechanism for reflexive shifts of attention, this result makes functional sense, given that many changes in posture will spatially realign tactile receptors relative to visual receptors, in a manner that can be indicated by proprioceptive inputs and, thus, compensated for. Given the suggested role for proprioception, we predict that deafferented patients with complete proprioceptive loss (see, e.g., Cole, 1995) should fail to show the remapping evidenced by the influence of current hand posture in our study.

Recent studies of neurological patients suffering from the attentional deficit of extinction after unilateral righthemisphere damage have uncovered visual-tactile spatial interactions that may relate to those found here in neurologically healthy participants. In a series of studies, di Pellegrino, Làdavas, and Farnè (1997; see also Làdavas, di Pellegrino, Farnè, \& Zeloni, 1998) and colleagues have shown that visual stimulation near the ipsilesional (right) hand can extinguish awareness of a touch on the contralesional (left) hand that would otherwise have been detected. This situation may be analogous, in some respects, to the opposite-side cue trials in the present ex- periments, in which a cue event in one modality (say, vision) near one hand leads to less efficient performance for a target event at the other hand in the other modality (touch, as in the present Experiment 1). Làdavas and colleagues have found that the extinction phenomenon in their patients can be modulated by current hand posture (e.g., with strong cross-modal extinction when the patient's right hand is placed near the right visual event, which is greatly reduced when the right hand is, instead, positioned out of view, behind the patient's back; see di Pellegrino et al., 1997; Làdavas et al., 1998). If these neurological phenomena are indeed related to the present findings in healthy participants, it should be possible to modulate the neurological results by manipulations of unseen hand posture (e.g., crossing of the hands), as was found here for normal tactile-visual links.

A possible neural mechanism for the spatial links between touch and vision revealed in both neurologically intact participants and in patients may involve populations of multimodal neurons, like those recently revealed in neurophysiological single-cell recording studies in animals. Cross-modal interactions have been revealed in many of the structures implicated in the direction of spatial attention (e.g., Andersen, Snyder, Bradley, \& Xing, 1997; Graziano \& Gross, 1994; Groh \& Sparks, 1996; Stein \& Meredith, 1993). For instance, multimodal neurons responding to both visual and tactile stimuli have now been reported subcortically, in the superior colliculus and the putamen (Graziano \& Gross, 1993), and cortically, in regions such as the parietal cortex (e.g., area 7b; Graziano \& Gross, 1994; Hyvärinen, 1981) and the premotor cortex (i.e., area 6; Graziano, Yap, \& Gross, 1994; Rizzolatti, Scandolara, Matelli, \& Gentilucci, 1981a, 1981b). Importantly, the receptive fields of such neurons are typically organized in close spatial register across the modalities, so that a similar region of space is responded to in both vision and touch by a given cell. Furthermore, the visual receptive fields of some of these cells (in parietal area $7 \mathrm{~b}$, inferior frontal area 6 , and the putamen) are apparently tied to the body part that contains the cell's tactile receptive field, following it around in space if that body part is moved, even when the eye is fixed (Graziano \& Gross, 1993, 1994). For example, such a neuron with a tactile receptive field on one hand will typically respond to visual events near that hand in external space, in such a manner that the responsive visual field actually shifts across the retina if hand posture is changed. Such effects can be found with passive movements performed under occlusion of the effective body part or in darkness (Graziano, Hu, \& Gross, 1997), thus implying a role for proprioceptive modulation, just as for the present cross-modal cuing effects in humans.

Such cell populations might enable tactile-visual links in exogenous spatial attention and also might allow these to remap across changes in posture, so as to retain crossmodal coordination with respect to external space. One can envisage how activation of a spatial subset of such a multimodal cell population by a cue stimulus in one 
modality at a particular location might then boost responses to a target event in the other modality at the same location, in a manner that could be modulated by proprioception, just as we found. Thus, a tactile cue (as in Experiment 2) might interface with visual processing by means of multimodal neurons that pool information across touch, vision, and proprioception.

However, cross-modal influences may not be restricted solely to multimodal structures but might also affect unimodal structures. Feedback from the activity produced at multimodal levels by the cue might be able to modulate initial responses to a target in a second modality, even at relatively early unimodal levels of sensory processing. Some preliminary evidence now exists for this possibility, based on recent $f M R I$ and ERP data. With ERP measures of voltage fluctuations at the scalp, it has been reported that a tactile cue can affect relatively early ERP components produced by a subsequent visual target (Kennett et al., 2001; see also McDonald \& Ward, 2000, for a related audiovisual study). In this research, the N1 visual component was observed to be larger for a visual target on the same side as the tactile cue, as compared with a target appearing on the opposite side. Since the N1 component is thought to reflect modality-specific visual processing in the extrastriate visual cortex (Mangun, 1995), this implies a multimodal influence on visual processing, via backprojections. Similarly, using fMRI, Macaluso, Frith, and Driver (2000) showed that a tactile cue to one hand (held in an uncrossed posture) can enhance responses in the visual cortex (the lingual gyrus) for a visual target at the same location in space. Moreover, with such multimodal stimulation at a common location, the visual cortex, and the somatosensory cortex became more strongly coupled with activity in the multimodal parietal cortex.

The present behavioral results suggest that it will be important to extend such neural measures in order to examine situations in which different unseen postures are adopted, thus addressing the neural basis of remapping for cross-modal attentional links. Our results indicate that tactile-visual links in exogenous covert spatial attention do not reflect a fixed mapping, whereby stimulation of one visual field will always lead to most efficient performance when paired with stimulation of one particular hand. Instead, the cross-modal cuing effects remap across changes in unseen posture, implying a modulatory role for proprioception.

\section{REFERENCES}

Andersen, R. A., Snyder, L. H., Bradley, D. C., \& Xing, J. (1997). Multimodal representation of space in the posterior parietal cortex and its use in planning movements. Annual Review of Neuroscience, 20, 303-330.

Bradshaw, J. L., Howard, M. J., Pierson, J. M., Phillips, J., \& BradSHAW, J. A. (1992). Effects of expectancy and attention in vibrotactile choice reaction-time tasks. Quarterly Journal of Experimental Psychology, 44A, 509-528.

Butter, C. M. (1987). Varieties of attention and disturbances of attention: A neuropsychological analysis. In M. Jeannerod (Ed.), Neurophysiological and neuropsychological aspects of spatial neglect (pp. 1-24). Amsterdam: North-Holland.
Butter, C. M., Buchtel, H. A., \& SAntucci, R. (1989). Spatial attentional shifts: Further evidence for the role of polysensory mechanisms using visual and tactile stimuli. Neuropsychologia, 27, 1231-1240.

Cole, J. O. (1995). Pride and a daily marathon. Cambridge, MA: MIT Press.

di Pellegrino, G., Làdavas, E., \& Farnè, A. (1997). Seeing where your hands are. Nature, $\mathbf{3 8 8}, 730$.

Downing, P. E., \& Treisman, A. M. (1997). The line-motion illusion: Attention or impletion? Journal of Experimental Psychology: Human Perception \& Performance, 23, 768-779.

Dufour, A. (1999). Importance of attentional mechanisms in audiovisual links. Experimental Brain Research, 126, 215-222.

Graziano, M. S. A., \& Gross, C. G. (1993). A bimodal map of space: Somatosensory receptive-fields in the macaque putamen with corresponding visual receptive-fields. Experimental Brain Research, 97, 96-109.

Graziano, M. S. A., \& Gross, C. G. (1994). The representation of extrapersonal space: A possible role for bimodal, visual-tactile neurons. In M. S. Gazzaniga (Ed.), The cognitive neurosciences (pp. 10211034). Cambridge, MA: MIT Press.

Graziano, M. S. A., Hu, X. T., \& Gross, C. G. (1997). Visuospatial properties of ventral premotor cortex. Journal of Neurophysiology, 77, 2268-2292.

Graziano, M. S. A., Yap, G. S., \& Gross, C. G. (1994). Coding of visual space by premotor neurons. Science, 266, 1054-1057.

Groh, J. M., \& Sparks, D. L. (1996). Saccades to somatosensory targets: 3. Eye-position-dependent somatosensory activity in primate superior colliculus. Journal of Neurophysiology, 75, 439-453.

Hikosaka, O., Miyauchi, S., Takeichi, H., \& Shimojo, S. (1996). Multimodal spatial attention visualized by motion illusion. In T. Inui \& J. L. McClelland (Eds.), Attention and performance XVI: Information integration in perception and communication (pp. 237-261). Cambridge, MA: MIT Press, Bradford Books.

Howell, D. C. (1997). Statistical methods for psychology (4th ed.). London: Duxbury.

HYvÄRINEN, J. (1981). Regional distribution of functions in parietal association area 7 of the monkey. Brain Research, 206, 287-303.

Kennett, S., Eimer, M., Spence, C., \& Driver, J. (2001). Tactile-visual links in exogenous spatial attention under different postures: Convergent evidence from psychophysics and ERPs. Journal of Cognitive Neuroscience, 13, 462-478.

Kinsbourne, M. (1975). The mechanism of hemispheric control of the lateral gradient of attention. In P. M. A. Rabbitt \& S. Dornic (Eds.), Attention and performance V (pp. 81-97). New York: Academic Press.

Kinsbourne, M. (1987). Mechanisms of unilateral neglect. In M. Jeannerod (Ed.), Neurophysiological and neuropsychological aspects of spatial neglect (pp. 69-86). Amsterdam: North-Holland.

KleIN, R., \& KerR, B. (1974). Visual signal detection and the locus of foreperiod effects. Memory \& Cognition, 2, 431-435.

Làdavas, E., di Pellegrino, G., Farnè, A., \& Zeloni, G. (1998). Neuropsychological evidence of an integrated visuotactile representation of peripersonal space in humans. Journal of Cognitive Neuroscience, 10, 581-589.

Macaluso, E., Frith, C. D., \& Driver, J. (2000). Modulation of human visual cortex by cross-modal spatial attention. Science, 289, 1206-1208.

MANGUN, G. R. (1995). Neural mechanisms of visual selective attention. Psychophysiology, 32, 4-18.

McDonald, J. J., Teder-Sälejärvi, W. A., Heraldez, D., \& HillYARD, S. (2001). Electrophysiological evidence for the "missing link" in cross-modal attention. Canadian Journal of Experimental Psychology, 55, 143-151.

McDonald, J. J., Teder-Sälejärvi, W. A., \& Hillyard, S. A. (2000). Involuntary orienting to sound improves visual perception. Nature, 407, 906-908.

McDonALD, J. J., \& WARD, L. M. (2000). Involuntary listening aids seeing: Evidence from human electrophysiology. Psychological Science, 11, 167-171.

Niemi, P., \& NÄ̈̈тÄNEN, R. (1981). Foreperiod and simple reaction time. Psychological Bulletin, 89, 133-162.

Posner, M. I., Klein, R., Summers, J., \& Buggie, S. (1973). On the selection of signals. Memory \& Cognition, 1, 2-12. 
Rafal, R., Henik, A., \& Smith, J. (1991). Extrageniculate contributions to reflex visual orienting in normal humans: A temporal hemifield advantage. Journal of Cognitive Neuroscience, 3, 322-328.

Riggio, L., Gawryszewski, L. D., \& Umiltà, C. (1986). What is crossed in crossed-hand effects? Acta Psychologica, 62, 89-100.

Rizzolatti, G., Scandolara, C., Matelli, M., \& Gentilucci, M. (1981a). Afferent properties of periarcuate neurons in macaque monkeys: 1. Somatosensory responses. Behavioural Brain Research, 2 , 125-146.

Rizzolatti, G., Scandolara, C., Matelli, M., \& Gentilucci, M. (1981b). Afferent properties of periarcuate neurons in macaque monkeys: 2. Visual responses. Behavioural Brain Research, 2, 147-163.

Robinson, D. L., Bowman, E. M., \& Kertzman, C. (1995). Covert orienting of attention in macaques: II. Contributions of parietal cortex. Journal of Neurophysiology, 74, 698-712.

Robinson, D. L., \& Kertzman, C. (1995). Covert orienting of attention in macaques: III. Contributions of the superior colliculus. Journal of Neurophysiology, 74, 713-721.

Simon, J. R. (1990). The effects of an irrelevant directional cue on human information processing. In R. W. Proctor \& T. G. Reeve (Eds.), Stimulus-response compatibility (pp. 31-86). Amsterdam: Elsevier.

SPENCE, C. (2001). Cross-modal attentional capture: A controversy resolved? In C. Folk \& B. Gibson (Eds.), Attention, distraction and action: Multiple perspectives on attentional capture (pp. 231-262). Amsterdam: Elsevier.

Spence, C., \& Driver, J. (1994). Covert spatial orienting in audition: Exogenous and endogenous mechanisms. Journal of Experimental Psychology: Human Perception \& Performance, 20, 555-574.

Spence, C., \& Driver, J. (1996). Audiovisual links in endogenous covert spatial attention. Journal of Experimental Psychology: Human Perception \& Performance, 22, 1005-1030.

Spence, C., \& Driver, J. (1997). Audiovisual links in exogenous covert spatial orienting. Perception \& Psychophysics, 59, 1-22.
Spence, C., Nicholls, M. E. R, Gillespie, N., \& Driver, J. (1998). Cross-modal links in exogenous covert spatial orienting between touch, audition, and vision. Perception \& Psychophysics, 60, 544-557.

Spence, C., Pavani, F., \& Driver, J. (2000). Crossmodal links between vision and touch in covert endogenous spatial attention. Journal of Experimental Psychology: Human Perception \& Performance, 26, 1298-1319.

Stein, B. E., \& Meredith, M. A. (1993). The merging of the senses. Cambridge, MA: MIT Press.

Steinman, S. B., \& Steinman, B. A. (1997). The line motion illusion: Visual attention or apparent motion? [Abstract]. Investigative Ophthalmology \& Visual Science, 38, 1748.

Umiltà, C., Rizzolatti, G., Anzola, G. P., Luppino, G., \& Porro, C. (1985). Evidence of interhemispheric transmission in laterality effects. Neuropsychologia, 23, 203-213.

Wallace, R. J. (1971). S-R compatibility and the idea of a response code. Journal of Experimental Psychology, 88, 354-360.

WALlace, R. J. (1972). Spatial S-R compatibility effects involving kinesthetic cues. Journal of Experimental Psychology, 93, 163-168.

WANN, J. P., \& IвRAHIM, S. F. (1992). Does limb proprioception drift? Experimental Brain Research, 91, 162-166.

\section{NOTE}

1. Although proprioception is likely to have informed the participants of their current posture, alternative amodal sources of information existed, since the participants were instructed where to place their arms at the start of experimental blocks.

(Manuscript received December 31, 2000; revision accepted for publication February 26, 2002.) 